\title{
A case of progressive aphasia without dementia: "temporal" Pick's disease?
}

\author{
Ph Scheltens, G J Hazenberg, J Lindeboom, J Valk, E Ch Wolters
}

\begin{abstract}
We report a patient who suffered from progressive aphasia for nine years, before developing mild behavioural disturbances. Sequential computed tomography (CT) scanning and magnetic resonance (MRI) imaging showed progressive bilateral temporal atrophy. The case is thought to be a temporal form of Pick's disease, in which isolated progressive aphasia was the only symptom over many years.
\end{abstract}

Progressive aphasia without dementia (PAWD) is recognised as a distinct syndrome, associated with left perisylvian degeneration. ${ }^{1}$ We present a patient with the clinical picture of PAWD, in which clinical, neuropsychological and neuroradiological data suggest bilateral temporal involvement and a temporal form of Pick's disease.

\section{Case Report}

A 59 year old right handed carpenter first visited a neurologist in 1984 five years after the onset of a slowly progressive language disorder which started with the misunderstanding of the spoken word and some spontaneous paraphasias. Neurological examination revealed no

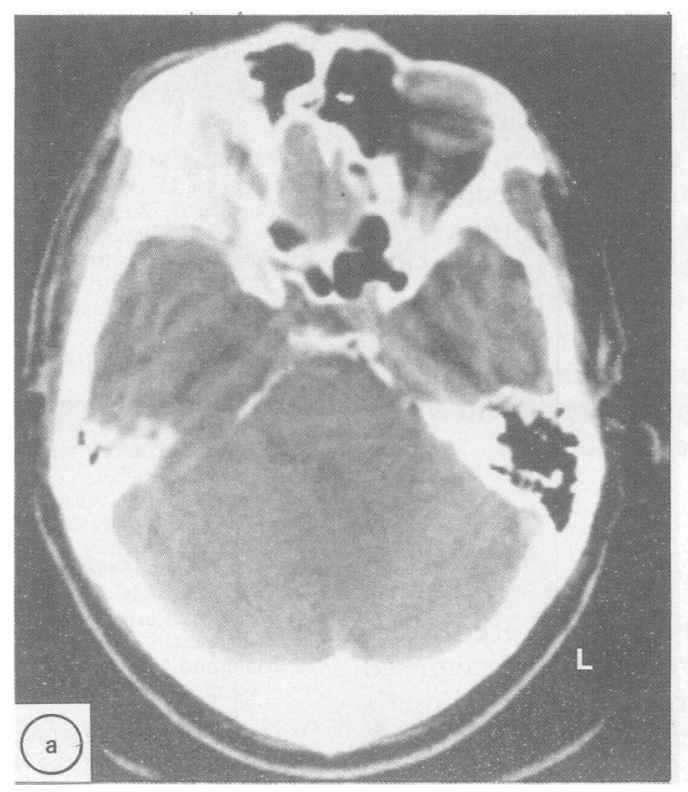

abnormalities. Electroencephalography (EEG) and computed tomography (CT) scans (fig 1A) were normal. Neuropsychological assessment showed impairment of naming and word finding. Digit span was reduced (5 forward, 4 backward). Nonverbal test performance was normal. No definite diagnosis was established at that time. Two years later he was referred to our outpatient clinic but was unable to communicate. He was, however, able to spend his time cycling around, without getting lost. $\mathrm{He}$ could still sing, in tune, complete operas from memory. No cognitive or personality changes were observed.

He reacted to environmental sounds, but hardly to words; a hearing aid had been prescribed without effect. Neurological examination revealed no abnormalities except severe Wernicke aphasia. Routine blood and spinal fluid tests were normal.

CT revealed enlargement of the inferior horns, predominantly on the left side (fig 1b). EEG showed normal alpha wave pattern without temporal slowing.

Neuropsychological assessment showed a further decline in the patient's ability to name objects and perform digit span (four forward, two backward). Word comprehension was disturbed. Nonverbal performance remained normal: Benton's Judgement of Line Orientation was above average and designs were copied

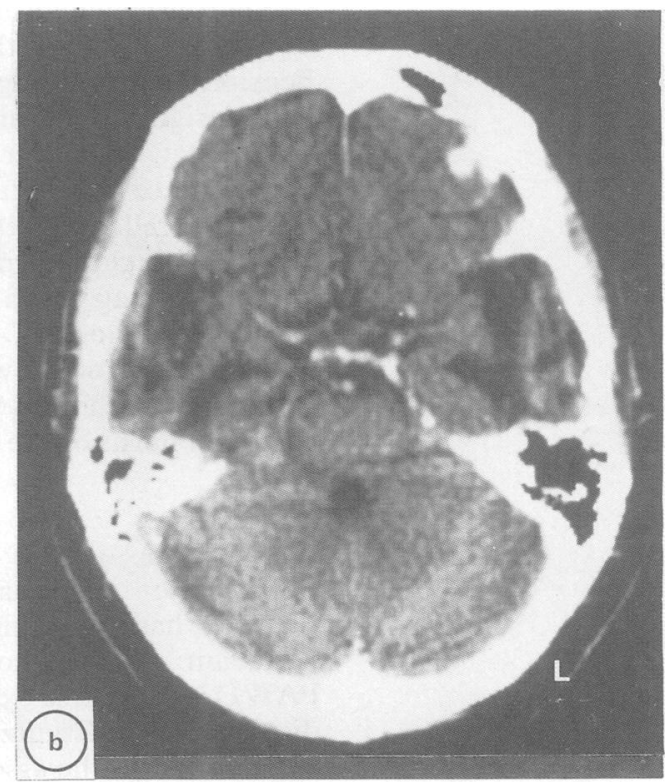

Figure $1 C T$ scans at the level of the inferior horns of the ventricles. A: no abnormalities (1984). B: moderate asymmetric enlargement of the inferior horns. (1986) $L=L$ eft. 
Figure 2 MR image 1988: at the level of the inferior horns with $T 1$ weighting: SE 350/22/4/ $192 \times 256$. Note the symmetric dilation of the inferior horns and severe atrophy of the temporal lobes. $L=L e f t$

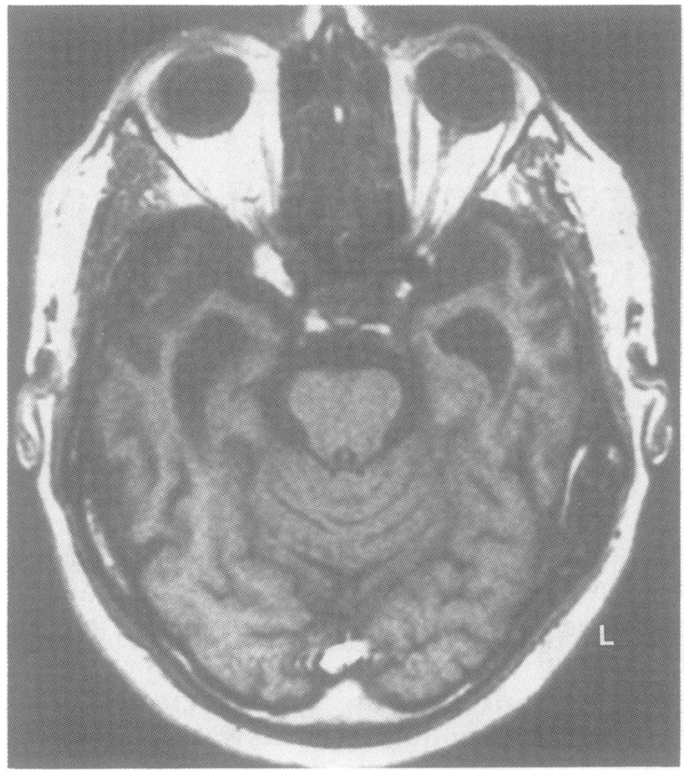

with great precision. The WISC-mazes were solved without errors.

Approximately two years later (nine years after onset of the first symptoms), spontaneous speech was fluent but meaningless with paraphasias and a tendency to perseverate. Auditory comprehension was impaired even at the single word level. Repetition of phrases, long sentences and words was sometimes paraphasic and perseverative. Reading aloud was intact without comprehension. Writing to dictation was impossible. Singing (his former hobby) had also become impaired. His appearance was neat and tidy, but a slight dressing apraxia was noted.

On his daily cycling tours he never lost his way, even in an unfamiliar environment. His conduct, however, was no longer socially acceptable. For instance, he would drive in the middle of the road shouting at cars that passed, and disregarding all traffic rules. Seizures, urine incontinence and sexual disinhibition were not observed.

Neuropsychological evaluation showed further progression of the naming and comprehension deficits. Judgement of Line Orientation, design copying and solution of mazes were still intact. However, there was a defective performance on Benton's Facial Recognition Test. Excellent preservation of nonverbal capacities including memory and topografical skills argued against a global mental decline.

Neuroradiological evaluation by CT and MRI (fig 2) now showed a marked atrophy of both temporal lobes, with enlargement of the temporal horns of the ventricles.

\section{Discussion}

Progressive aphasia without dementia (PAWD) has been defined as a clinical entity by Mesulam. ${ }^{1}$ Others, however, have recognised PAWD as the first expression of Alzheimer's disease or even Creutzfeld-Jakob's disease. ${ }^{234}$

Attention to the association with Pick's disease has been given by Holland et $a l^{5}$ who described a patient experiencing an isolated progressive aphasia for 12 years. Necropsy showed evidence of Pick's disease. The case described by Wechsler et al, ${ }^{6}$ which proved to be Pick's disease, can also be regarded as an example of PAWD although the patient was demented two years after onset of the symptoms. Van Mansvelt, ${ }^{7}$ in his study of 171 cases of Pick's disease found 29 cases with predominantly temporal lobe involvement. These cases often presented with Wernicke's aphasia long before personality changes occurred. Asymmetric temporal lobe involvement, predominantly affecting the left hemisphere, was present in half the cases. His descriptions of the "temporal" cases strongly resemble PAWD. Malamud and Boyd ${ }^{8}$ described a patient with progressive anomia and preservation of memory and orientation, who at necropsy showed severe atrophy of both temporal lobes and changes typical of Pick's disease.

In our case striking atrophy of both temporal lobes was recognised on MRI and CT nine years after onset of the symptoms. Neuroradiological signs, described as "typical" for Pick's disease were not seen. Such descriptions, however, mainly refer to the frontal type of Pick's disease. ${ }^{9}$ Right temporal lobe involvement was first suspected when the performance on Benton's Facial Recognition Test became poor, even though, aphasic patients with comprehension deficits may score the same levels. ${ }^{10}$

EEG examinations continued to remain normal in our patient. This nearly always rules out the existence of Alzheimer's disease, but is compatible with Pick's disease. In reports of PAWD thus far EEG's have been normal or have showed only left hemisphere slowing.

We feel that our case may represent a temporal lobe variant of Pick's disease, in which slowly progressive aphasia without dementia preceded mild personality changes for nine years. In addition, we think that PAWD should be considered as a presenting symptom of Pick's disease, Alzheimer's disease, or Creutzfeld-Jakob's disease until a specific pathoanatomical substrate has been defined, which justifies the existence of PAWD as a clinical entity.

1 Mesulam MM. Slowly Progressive Aphasia Without Generalised Dementia. Ann Neurol 1982;11:592-8.

2 Pogacar S, Williams RS. Alzheimer's disease presenting as slowly progressive aphasia. RI Med J 1984;67:181-5.

3 Poeck K, Luzatti C. Slowly progressive aphasia in three patients: The problem of accompanying neuropsychological deficit. Brain 1988;111:151-68.

4 Mandell AM, Alexander MP, Carpenter S. CreutzfeldtJakob disease presenting as isolated aphasia. Neurology 1989;39:55-8.

5 Holland AL, McBurney DH, Moossy J, Reinmuth OM. The dissolution of language in Pick's disease with neurofibrillary tangles: a case study. Brain Lang 1985;24:36-58.

6 Wechsler AF, Verity MA, Rosenschein S, Fried I, Scheibel AB. Pick's disease. A clinical, computed tomographic, and histologic study with golgi impregnation observations. histologic study with golgi im
Arch Neurol 1982;39:287-90.

7 Van Mansvelt J. Pick's Disease. A syndrome of lobar cerebral atrophy. Vd Loeff, Enschede 1954. Thesis, Utrecht.

8 Malamud N, Boyd Jr A. Pick's disease with atrophy of the temporal lobes: a clinicopathologic study. Arch Neurol Psych 1940;43:210-22.

9 Groen JJ, Hekster REM. Computed tomography in Pick's disease: findings in a family affected in three consecutive generations. J Comput Assist Tomogr 1982;6:907-11.

10 Hamsher K, Levin HS, Benton AL. Facial recognition in patients with focal brain lesions. Arch Neurol 1979; 36:837-9. 